\title{
Precision sculptured surface CNC machining using cutter location data
}

\author{
Nikolaos A. Fountas ${ }^{1, \text { a }}$ Nikolaos M. Vaxevanidis ${ }^{2, b^{*}}$ Constantinos I. Stergiou $^{3, c}$ \\ and Redha Benhadj-Djilali ${ }^{4, d}$ \\ ${ }^{1,2}$ Laboratory of Manufacturing Processes and Machine Tools (LMProMaT), Department of \\ Mechanical Engineering Educators, School of Pedagogical and Technological Education (ASPETE), \\ GR 14121, N. Heraklion Attikis, Athens, Greece. \\ ${ }^{3}$ Department of Mechanical Engineering, Piraeus University of Applied Sciences, GR 12244, \\ Egaleo, Athens, Greece. \\ ${ }^{4}$ Faculty of Science, Engineering and Computing (SEC), Roehampton Vale Campus, Kingston \\ University, Friars Avenue, Kingston Upon Thames, SW15 3DW London, UK.

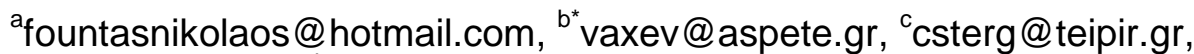 \\ ${ }^{\mathrm{d}}$ R.Benhand-Djilali@kingston.ac.uk
}

Keywords: Sculptured surfaces. CNC machining, Cutter location data, Machining error.

\begin{abstract}
Industrial parts with sculptured surfaces are typically, manufactured with the use of CNC machining technology and CAM software to generate surface tool paths. To assess tool paths computed for 3- and 5-axis machining, the machining error is evaluated in advance referring to the parameter controlling the linearization of high-order curves, as well as the scallop yielded as a function of radial cutting engagement parameter. The two parameters responsible for the machining error are modeled and corresponding cutter location data for tool paths are utilized to compare actual trajectories with theoretical curves on a sculptured surface (SS) assessing thus the deviation when virtual tools are employed to maintain low cost; whilst ensuring high precision cutting. This operation is supported by applying a flexible automation code capable of computing the tool path; extracting its CL data; importing them to the CAD part and finally projecting them onto the part's surface. For a given tolerance, heights from projected instances are computed for tool paths created by changing the parameters under a cutting strategy, towards the identification of the optimum tool path. To represent a global solution rough machining is also discussed prior to finish machining where the new proposals are mainly applied.
\end{abstract}

\section{Introduction}

Sculptured surface machining (SSM) [1] is a fundamental manufacturing process in mechanical industy. As a special technology it is; it employs cutting edge resources to design, test and machine free-form parts to meet functionality and aesthetics. Such resources involve advanced systems such as computer-aided manufacturing (CAM) software for machining modeling and multi axis surface machining via computer numerical control (CNC) machine tools. In SSM, complex shapes and profiles are represented in parametric forms such as Bezier, B-Spline and NURBS. As a result, cutting-edge CNC systems implement parametric interpolators to accurately create cutting paths without segmentation contour processing. Parametric interpolators manage to overcome problems in real applications such as huge amounts of NC data, unsmooth motion speed, surface accuracy deterioration, machine vibrations [2,3], etc. Although the efficiency of parametric interpolators is at its edge, only a group of CNC units support such functions whilst they are provided as additional modules with independent policy to the rest of the systems. With the rapid advances in software and 
hardware, large data files and data processing are of minor concern. High frequency servo loop functions and microprocessors enable good transitions during the cutting process while coping with large NC files. It is also evident that the majority of modern machine shops worldwide still employ linear-circular interpolators to meet customers' demands as far as precision is concerned. Since there is still room for the applications of conventional interpolators there is also the need to control their machining error yielded when implementing them to high-precision machining.

A large body of contributions has already introduced several techniques to control the machining error. As an importance instance, cutter location file (CL Data) file has received extensive attention for the proposals of these research works. In [4] a bi-arc curve fitting approach to efficiently interpolate complex surfaces is proposed. The distances computed from the tessellation of the surface through bi-arcs utilize the CL data. In [5] a methodology to investigate the geometrical machining error for large sculptured parts by employing 5-axis machining is proposed. In their work a mathematical expression is used as a function to be solved where the step formulated by two successive cutter positions is controlled via chordal deviation. An interpolation scheme is presented; based on adaptive feedrate to machine sculptured surfaces in [6]. Drysdale et al., [7] computed the minimum number of circular arcs/bi-arcs needed to approximate curves by setting three criteria. The first criterion is the positions (start and end) of the arcs; the second is the correct side that arc segments should be placed and the third is the non-intersection between the arcs and chordal deviation. In [8] Vijayaraghavan et al., identified some of the drawbacks when employing NURBS interpolation and contributed to precision machining operations by presenting a method of subdividing curves via the application of "Catmull-Clark" subdivision scheme in two-dimensions to represent machine tool trajectories. Their goal was to find a set of points separated by the required curve's chord length. This set of points involves the CL data. In [9] a two-phase strategy is presented dealing with a 5-axis surface error optimization method. The first phase employs a module that computes parametric intervals via the $2^{\text {nd }}$ Taylor's expansion whilst chord error evaluation is achieved in the second phase via CL data-based cutting simulations.

\section{Machining objectives}

Objectives for optimizing surface machining are defined according to the process planning phase being referred. Rough machining assessment can be conducted by specifying targets such as material removal rate (MRR); machining time or cycle time and remained volume on the ideal part's surface. On the contrary machining error, geometrical and dimensional accuracy as well as tolerancing are key attributes for evaluation when it comes to finish machining. The curent study seeks to model roughing and finishing operations with the use of programmatical objects that directly related to these objectives so as to optimize them via artificial intelligence techniques or any other of the available methods in the literature.

Rough machining toolpaths are applied first to remove the main stock volume and leave a much more regular shape for the machining operations which follow. The part's surface after roughing consists of a number of scallops of either equal or different heights formulating the volume to be removed by finishing. In 3-axis finishing the cutting tool contacts the roughed steps with its axis always normal to part's surface (Fig.1a) whereas in 5-axis finishing the tool meets differently the remaining volume represented by the scallops according its inclination with reference to the part's surface. One can compute the remaining material's height by projecting vertical lines from the ideal part onto the roughed part (Fig.1b). It is essential that a small variance of scallop heights ought to be kept so as to achieve low cutting force variation. 


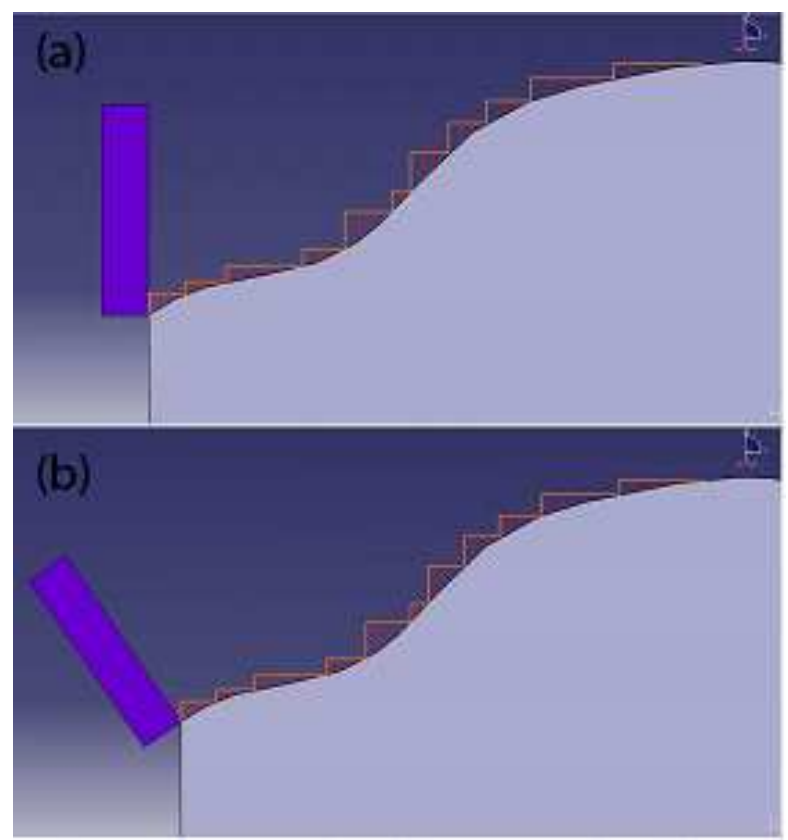

Fig.1: Typical finishing strategies of a roughed sculptured part: (a) 3-axis; (b) 5-axis.

A double-bounded objective is studied; allowing the comparison of several toolpath trajectories for roughing. The objective involves two criteria namely remaining volume and machining time which both tend to force CAM software to produce a rough cutting style capable of producing uniformly distributed magnitudes of Z-level passes. Note that remaining volume and machining time are two criteria opposed to each other since less remaining material equals to more (smaller) scallops whilst less machining time equals to less (larger) scallops. Having such a trade-off exploited in an optimization algorithm the benefit would be an optimum result satisfying both criteria simultaneously, which is; producing a toolpath with equidistant roughing levels via the control of maximum stepdown parameter and radial engagement of the tool when penetrating the material (stepover). In other words such a target would produce an iso-scalloped rough machining an operation deeded as the optimum by several researchers [10-12]. What gives this target its added value in the filed of multi-objective CNC machining optimization is the capability of controlling toolpath efficiency through stochastic assessment techniques rather that computing by numerical methods or utilizing analytical models for prediction.

The study employs CAM software automation routines developed by taking advantage of the interface provided for customizing the virtual manufacturing environment. After the machining modelling operation the history tree is programmatically retrieved and the corresponding computed toolpath is tracked. Further on, machining time calculated for that toolpath is extracted and stored as an output. With reference to stepover, stepdown and feed rate values, the removed material for each roughing pass can be computed as follows:

$V_{\text {removed }}=a_{p} \times w \times V_{f}$

where,

$a_{p}$ : the stepdown value for each pass in $(\mathrm{mm})$;

$w$ : the stepover value for each pass in $(\mathrm{mm})$;

$V_{f}$ : the cutting feed for each pass in $(\mathrm{mm} / \mathrm{min})$. 
CAM software is capable of extracting machining and cycle time with the former to represent the exact cutting time and the latter to include idle time as well. Machining time is used for the identification of the total cutting length towards the calculation of remaining volume after roughing. In general machining time is given as:

$t_{m}=\frac{L_{T O T}}{V_{f}}$

where $L_{T O T}$ corresponds to the total length to be machined and computed by taking into account cutting tool's geometrical configurations, radial-axial cutting depths and start-end cutting positions (Fig.2) and for these positions $L_{c}$ is computed as $L_{c}=\sqrt{w(D-w)}$

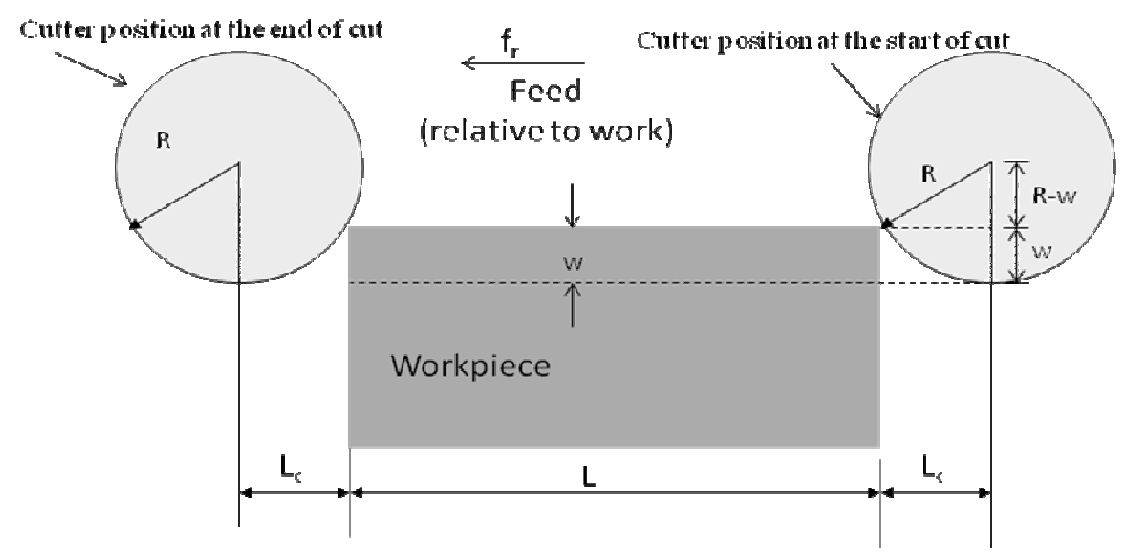

Fig.2: Top view of end milling operation and machining distance computation.

Thus, the material remaining for finishing will be given by subtracting the ideal part's volume and removed volume by the volume of the stock material as follows:

$V_{\text {remained }}=V_{\text {stock }}-V_{\text {removed }}-V_{\text {ideal_part }}$

Primary finish machining objectives involve part quality, dimensional accuracy and tolerance. When it comes to CAM software applications for machining modeling, finishing strategies are applied to generate regular tool paths that will follow the part's sculptured surface (SS) under a given tolerance with reference to the cutting tool's configurations and the surface's varying curvature. As a final output CAM software generates the cutter location points (CL Data). Given the set of CL data, a continous tool-path should be interpolated in order to specify the trajectory formulated by the discrete $\mathrm{CL}$ points. Thereby, precision machining is achieved if machining error is constrained within acceptable bounds during interpolation.

The ongoing evolution of CNC control systems leads to the implementation of advanced interpolations to machine sculptured surfaces (SS) such as Non-uniform rational B-Splines (NURBS). NURBS can be employed when retrieving the high-precision contour control mode. The NURBS interpolation function can be then programmed according to ISO $6983 \mathrm{NC}$ code (G-code programming principles) and the specific NC control unit configurations. The function is actually a modal code of G-code group 01 and operates with the curve order $(\mathrm{P})$, the control points $(\mathrm{X}, \mathrm{Y}, \mathrm{Z})$, the weights (R), the knots (K) and the feedrate in $\mathrm{mm} / \mathrm{min}$. Despite the availability of such technology 
when it comes to CNC sculptured surface machining, its implementation seems to lag behind as it is still in its infancy [13]. Very few top CNC units apply NURBS interpolation and it is questionable whether NURBS interpolation indeed provides higher surface finish. Practically speaking, "segmented" interpolations (linear or circular) under a specified machining error may hold tolerances just precise as NURBS interpolations do with the difference that huge NC files need to be stored in NC control units against NURBS format which is by far more efficient. From a practical viewpoint, there is no accuracy level that NURBS interpolation can achieve whilst chordal interpolations cannot. To do so, numerous NC blocks are resulted increasing the NC program's length, yet; this is not of major concern given the current state of advances in machine tools and CNC programming units. Fortunately, high-tech NC systems have become more sophisticated and "adept" while coping with large NC files, under fast processing rates. Moreover, high frequency servo loop functions integrated to CNC systems, allow smoother machining operations whilst maintaining good transition from one move to the next, in terms of feed rate. These aspects lead to reconsider the application of common interpolators when it comes to high-precision machining operations [14].

Machining error directly affects the overall shape fidelity and surface accuracy. In a software-based environment machining error ought to be studied by process parameters that hold dominant influence onto a sculptured part's surface. Most important parameters identified as being responsible for the machining error are the maximum discretization step and scallop height. The former parameter is deals with the length of the linear segments iterpolating the curve controlling thus the longitudinal step on the feed direction whereas the latter expresses the excess material left in the transversal direction on successful machining pass intervals. As a consequence, two form errors occur during toolpath interpolation owing to these two directions. The first type of error is known as the chord error and is the maximum distance between a linear segment (chord) connecting two consecutive interpolated points and the corresponding arc on the curve (Fig. 3). The second type of error is yielded owing to the uncut volume left among the pairs of adjacent radial passes whilst it is created by the finite pick offset among the successive cutting paths.

(a)

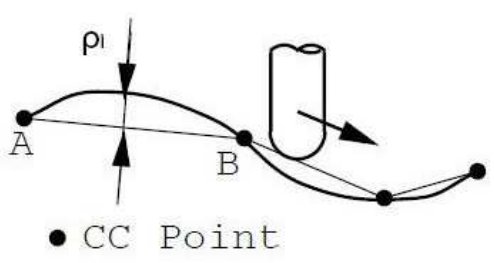

(b)

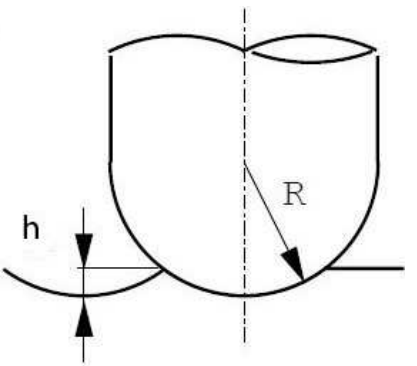

Fig.3: Machining parameters and objectives constituting the machining error (a) chord error; $(b)$ scallop height.

New criteria are introduced in the case of 3- and 5-axis enabling the indirect, yet; accurate control of both discretization step for curve segmentation and scallop height for the radial pass intervals. The criterion enabling a stochastic control of chord error is based on a mechanism that projects computed CL points formulating a toolpath so that maximum discretization step values produce resulting segmentation within tolerance. This is achieved by sequentially evaluating the differences among successful projection heights. The result is uniform patterns of cutter contact (CC) points - or identically cutter location (CL) points -, meaning effective tool trajectories, thus; regular toolpaths. Fig.4 illustrates the proposed technique and its impact to the chord error when it comes to the control of proper curve segmentation. 

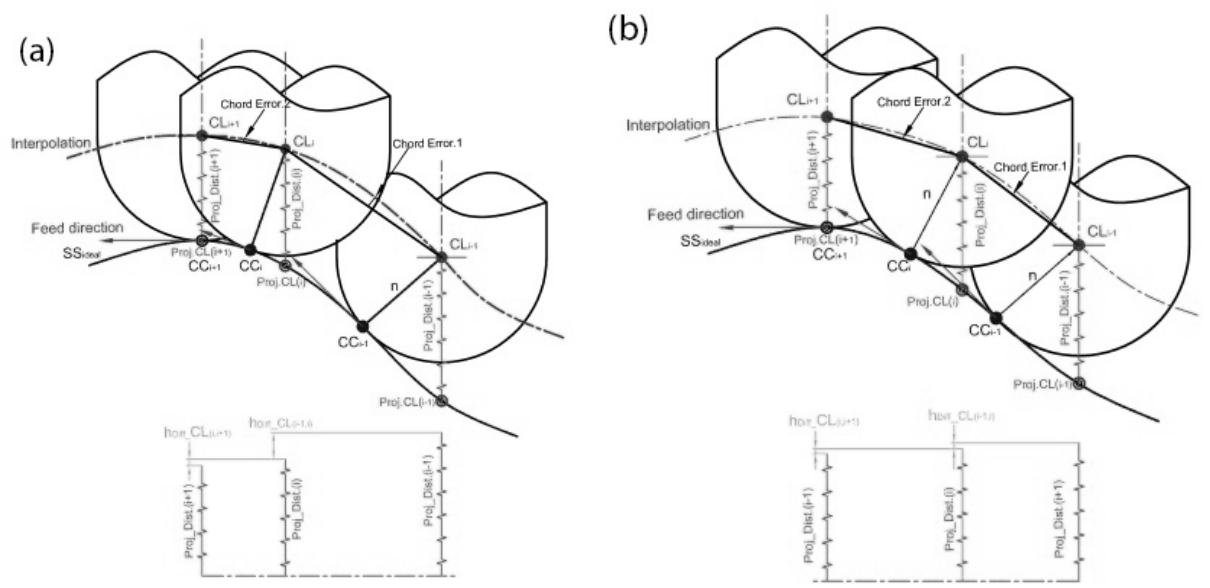

Fig.4: Chord error control: (a) conventional toolpath segmentation; (b) controlled toolpath segmentation via the proposed strategy.

Let the proposed optimization criterion to be indicated as $h_{C L i_{-} P j}^{D i f}$, stemming from the requirement of minimum incremental differences for all CL points that constituting the computed tool path. Hence,

$h_{C L i_{-} P j}^{D i f}=\left|h_{C L_{-} P j}(i-1)-h_{C L_{-} P j}(i)\right|=\min , \forall C C_{i} \in S S$

with,

$$
\sum_{C C_{i=1}}^{k} h_{C L i_{-} P j}=\min
$$

Where,

$h_{C L i_{-} P j}^{D i f}$ : The mean average of the differences computed when projecting CL points onto a part's sculptured surface to control discretization step and thus; chordal deviation, in $(\mathrm{mm})$;

$h_{C L_{-} P j}(i-1)$ : The projection height distance from the CL $(i-1)$ point to surface;

$h_{C L_{-} P j}(i)$ : The projection height distance from the CL $(i)$ point to surface.

Scallop height - which is also important for the overall representation of machining error-, can be modelled by taking into account the tool diameter and the effective tool diameter which is actually the true ball-end tool section in contact with the material. Should the two cutting depths (axial and radial) are taken into consideration (Fig.5), the scallop can be computed as follows:

$$
D_{E F F}^{3 x}=2 \times\left(\sqrt{\left(\frac{D}{2}\right)^{2}-\left(\frac{D}{2}-a_{p}\right)^{2}}\right.
$$

and finally, 
$h_{s}^{3 x}=\frac{D_{E F F}^{3 x}}{2}-\sqrt{\left(\frac{D_{E F F}^{3 x}}{2}\right)^{2}-\left(\frac{a_{e}}{2}\right)^{2}}$

where,

$D: \quad$ The tool diameter $(\mathrm{mm})$;

$D_{E F F}^{3 x}: \quad$ The effective cutter radius;

$h_{s}^{3 x}: \quad \quad$ The scallop height for 3-axis machining $\quad(\mathrm{mm})$;

$a_{e}: \quad$ The tool's stepover in the transversal direction $\quad(\mathrm{mm})$.

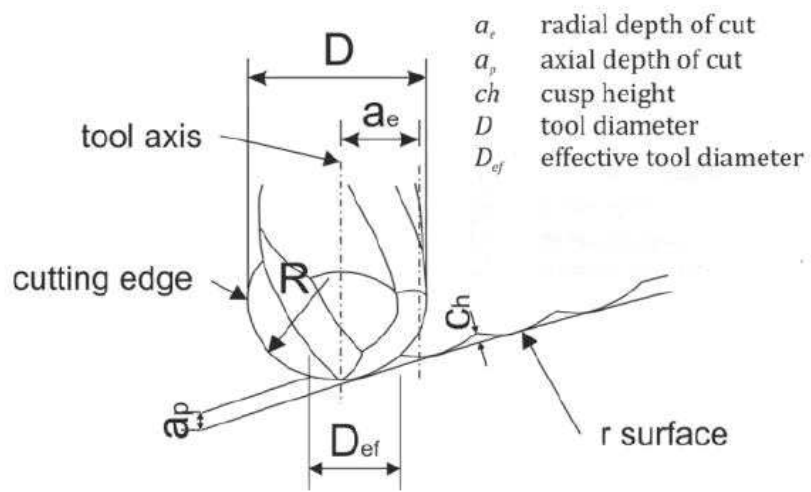

Fig.5: Scallop height representation and basic parameters.

The concept of the proposed criterion is slightly differenct in the case of 5-axis surface machining owing to its special ability of having an inclined tool following the curvature of a free-form part. In 5-axis machining mode the tool axis orientation is controlled through lead and tilt inclination angles. Lead angle $\left(\mathrm{a}_{\mathrm{L}}\right.$ or $\alpha$ ) controls the tool axis orientation which is normal to the part's surface in the feed direction, whilst tilt angle $\left(\mathrm{a}_{\mathrm{T}}\right.$ or $\beta$ ) inclines the tool axis in the perpendicular direction of feed. A common problem in 5-axis surface machining is how to restrict variations in terms of tool inclination so that collisions are avoided. As a result the philosophy of implementing the proposed machining error approach should definitely involve tool inclination and cope with the variable tool orientations to estimate accurate discretization step towards the feed direction given the tool type and the surface curvature as well. Kinematics of 5-axis surface machining suggest that each cutter location (CL) point is computed according to each of the corresponded cutter contact (CC) points, and a vector " $n$ " that is always normal to surface curvature (Fig.6).

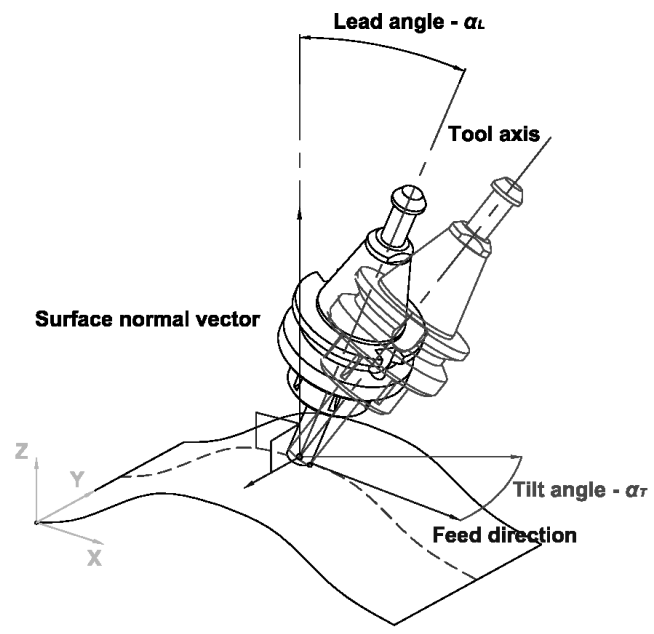

Fig.6: Cutting tool inclination in the case of 5-axis surface machining. 
Owing to the ability of the cutting tool to change its vertical axis orientation via inclination, projected CL points may produce low distances in advance, yet discretization step towards the path may not generate the required chord error, but a larger one. To cope with this problem in the 5-axis surface machining mode, the objective of attaining minimum incremental differences for all CL points formulating the tool path, is replaced here by a criterion aiming at controlling the ratio of normal vector magnitude when inclining the tool, to the projection distance of its given CL point on the surface. The proposed criterion is expressed as follows:

$R_{h_{C L i} \text { P }_{j}}^{n_{C i}}=\frac{\|\vec{n}\|_{C C i}}{h_{C L i} P j} \forall C C i \in S S, h_{C L i} P j \neq 0,\|\vec{n}\|>h_{C L i} P j$

where,

$R_{h_{C L_{-}-P j}}^{n_{C C i}}: \quad$ The ratio of normal vector magnitude to the distance of a projected CL point to control discretization step parameter and thus control chordal deviation $(\mathrm{mm})$;

$\|\vec{n}\|_{C C i}: \quad$ The normal vector magnitude at the $\mathrm{i}_{\mathrm{th}} \mathrm{CC}$ point;

$h_{C L i} P j: \quad$ The projection height distance from the $\mathrm{i}_{\text {th }}$ CL point's origin to sculptured surface.

Scallop height representation considers the different types of cutting tools and their capability to be inclined to sculptured surfaces. Having the same criterion in the case of 3-axis milling mode, focus is given when machining in 5-axis using flat-end and/or toroidal (bull) end-mills according to the surface normal vector as a key attribute related to their inclination angle for given cutter contact (CC) points for the toolpaths (Fig.7).

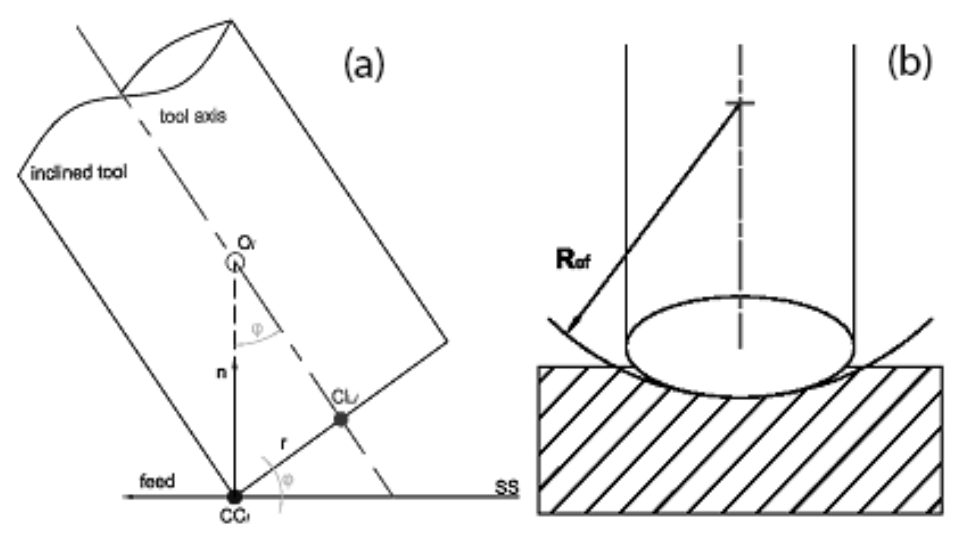

Fig.7: Cutting tool inclination in the case of 5-axis surface machining.

The shape of a flat-end mill engaging the surface is an elliptical silhouette when projected onto a plane perpendicular to the feed direction involving the surface normal. Thereby, at the corresponding CC point, the elliptical contour can be interpolated by a circle whose radius is known as the effective radius, $R_{e f}$. The effective radius is a function of both the tool's lead angle $a_{L}$ and its radius $R$, related as follows: 
$R_{e f}=\left\{\begin{array}{c}\frac{R_{\text {tool }}}{\sin \left(a_{L}\right)} \\ \frac{(R-r) \cos ^{2}\left(a_{L}\right)}{\sin \left(a_{T}\right)\left[1-\sin ^{2}\left(a_{T}\right) \times \sin ^{2}\left(a_{T}\right)\right]}+r\end{array}\right\}$

where,

$R_{e f}: \quad \quad \quad \quad$ The effective radius of the inclined tool when machining in 5-axis $(\mathrm{mm})$;

$R_{\text {tool }}: \quad$ The radius of the tool (either in $\mathrm{mm}$, or in $\% D$ );

$r: \quad$ The corner radius for a filleted-end (toroidal) mill, $(\mathrm{mm})$;

$a_{L}($ or $\alpha)$ : The tool's lead angle towards its path on the feed direction $\left({ }^{\circ} \mathrm{deg}\right)$;

$a_{T}($ or $\beta)$ : The tool's tilt angle $\left({ }^{\circ} \mathrm{deg}\right)$.

By varying lead angle $a_{L}$ and tilt angle $a_{T}$, a range of effective radii will be obtained and thus different scallops will occur.

\section{Application perspectives}

The models presented for machining error representation can be applied to an artificial intelligent algorithm towards the optimum results concerning all the machining parameters responsible to control the objectives this study discussed. Towards this potential, the authors look further ahead to extend their research efforts and implement their criteria to an intelligent system capable of ending up with a generic decision-making depending on the application whilst providing promising applicable results.

\section{Conclusions}

This paper presented a set of quality objectives so as to express the machining error in the special case of sculptured surface machining. Chord error and scallop magnitude are discussed as key attributes to precision cutting and surface accuracy whilst they are indirectly yet; effectively controlled via CAM software automation routines by taking advantage of the programming interface. Preliminary practical experiemnts have shown that the proposed expression for the models developed can be deemed trustworthy for repetitive evaluations towards the optimum results for machining parameters. 


\section{References}

[1] B.K. Choi and R.B. Jerard, Sculptured Surface Machining: Theory and Applications, Kluwer Academic Publishers, Dordrecht, 1998.

[2] E. Budak, L. T. Tunç, S. Alan and H. N. Özgüven, Prediction of Workpiece Dynamics and its Effects on Chatter Stability in Milling, CIRP An. Manuf. Technol. 61(1) (2012) 339-342.

[3] J. Yang and Y. Altintas, A generalized on-line estimation and control of five-axis contouring errors of CNC machine tools, Int. J. Mach. Tools Manuf. 88 (2015) 9-23.

[4] X. Yang, Efficient circular arc interpolation based on active tolerance control, Comput. Aided Des. 34 (2002) 1037-1046.

[5] X.-D. Lai, Y.-F. Zhou, J. Zhou, F.-Y. Peng and S.-J. Yan, Geometrical Error Analysis and Control for 5-axis Machining of Large sculptured surfaces, Int. J. Adv. Manuf Technol. 21 (2003) 110-118.

[6] S.S. Yen and P. L. Hsu, Adaptive feedrate interpolation for parametric curves with confined chord error, Comput. Aided Des. 34 (2002) 229-237.

[7] S. R. L. Drysdale, G. Rote and A. Sturm, Approximation of an open polygonal curve with a minimum number of circular arcs and biarcs, Computat. Geom. 41 (2008) 31-47.

[8] A. Vijayaraghavan, A. Sodemann, A. Hoover, J. R. Mayor and D. Dornfeld, Trajectory generation in high-speed, highprecision micromilling using subdivision curves, Int. J. Mach. Tools Manuf. 50(4) (2010) 394-403.

[9] Z. Lin, J. Fu, H. Shen and W. Gan, An accurate surface error optimization for five-axis machining of freeform surfaces, Int. J. Adv. Manuf. Technol. 71(5-8) (2014) 1175-1185.

[10] A. Can and A. Ünüvar, A novel iso-scallop tool-path generation for efficient five-axis machining of free-form surfaces, Int. J. Adv. Manuf. Technol. 51(9-12) (2010) 1083-1098.

[11] C. Min and X.-S. Gao, Iso-scallop tool-path generation of five-axis computer numerically controlled machining for cyclide splines, Proc. Inst. Mech. Eng. Part B: J. Eng. Manuf. 229(7) (2015) 1144-1156.

[12] A. A. Duroobi, J. H. Mohamed, B. I. Kazem and C. Wenlaing, Pick-Interval Scallop Height Estimation Using Three Types of Geometrical End Mill Cutters on CNC Milling Machine, Int. J. Eng. Technol. 31a (8) (2013) 1580-1591.

[13] M. Liu, Y. Huang, L. Yin, J. Guo, X. Shao and G. Zhang, Development and implementation of a NURBS interpolator with smooth feedrate scheduling for CNC machine tools, Int. J. Mach. Tools Manuf. 87 (2014) 1-15.

[14] C.-H. Chu, P.-H. Wu and W.-T. Lei, Tool path planning for 5-axis flank milling of ruled surfaces considering CNC linear interpolation, J. Intell. Manuf. 23 (2012) 471-480. 